\title{
From Sustainable Mobility to Good Deeds: Supporting School Participation during COVID-19 Emergency through a Playful Education Platform
}

\author{
Annapaola Marconi \\ marconi@fbk.eu \\ Fondazione Bruno Kessler \\ Trento, Italy
}

\author{
Gianluca Schiavo \\ gschiavo@fbk.eu \\ Fondazione Bruno Kessler \\ Trento, Italy
}

\author{
Paolo Massa \\ massa@fbk.eu \\ Fondazione Bruno Kessler \\ Trento, Italy
}

\author{
Eleonora Mencarini \\ mencarini@fbk.eu \\ Fondazione Bruno Kessler \\ Trento, Italy
}

\author{
Giulia Deppieri \\ gdeppieri@fbk.eu \\ Fondazione Bruno Kessler \\ Trento, Italy
}

\begin{abstract}
The COVID-19 emergency has posed many challenges to the worldwide school system leading to school closures and massive adoption of distance education. These events affected not only the way education is delivered but also the overall school community's wellbeing and cohesion. In this paper, we present a playful education platform originally designed to promote sustainable mobility in schools that has been adapted to support distance learning and community engagement during the health emergency. In the original version of the platform, the home-school distances traveled by students with sustainable means contributed to the advancement of the whole class in a collective virtual trip associated with playful learning material. Conversely, in a revised version of the platform adapted to the new context, students could advance in the virtual trip by reporting the good deeds performed at home. Findings from 161 questionnaires completed by teachers, families, and students, as well as results from online workshops with students, and the analysis of $\log$ files at the end of the school year revealed the opportunities and limitations of converting a playful activity conceived for being experienced in class into an online activity to be completed at home. The results show that the adapted version contributed to the school community cohesion and provided a positive experience to all its members, even though it was not able to completely overcome the generalized feeling of isolation. This paper contributes to understanding the social value of collaborative and playful digital activities that involve the entire school community, showing how technology can impact education and support communities in times of crisis.
\end{abstract}

\section{CCS CONCEPTS}

- Human-centered computing $\rightarrow$ Collaborative and social computing systems and tools.

\section{cc) (†)(2)}

This work is licensed under a Creative Commons

Attribution-NonCommercial-ShareAlike International 4.0 License.

IDC '21, fune 24-30, 2021, Athens, Greece

(c) 2021 Copyright held by the owner/author(s).

ACM ISBN 978-1-4503-8452-0/21/06.

https://doi.org/10.1145/3459990.3460718

\section{KEYWORDS}

Playful education, Sustainability, COVID-19, Distance learning

ACM Reference Format:

Annapaola Marconi, Gianluca Schiavo, Paolo Massa, Eleonora Mencarini, and Giulia Deppieri. 2021. From Sustainable Mobility to Good Deeds: Supporting School Participation during COVID-19 Emergency through a Playful Education Platform. In Interaction Design and Children (IDC '21), fune 24-30, 2021, Athens, Greece. ACM, New York, NY, USA, 7 pages. https: //doi.org/10.1145/3459990.3460718

\section{INTRODUCTION}

The COVID-19 health emergency of 2020 has posed unique challenges to the school system leading to worldwide school closures and rapid transition to distance learning. The COVID-19 restrictions have had an impact not only on the way education is delivered but also on the school community's wellbeing and cohesion [17]. The emergency has indeed urged school communities to rethink and reorganize themselves in a short time through distance learning and ultimately overcome the impacts of such difficult events [18]. In this respect, community-centered technology has proved handy in responding to the COVID-19 emergency, not only supporting communities with technological solutions [2, 4], but also enabling and enhancing collaboration and networking within communities [9]. This is even more relevant in the school context, where peer support and collaboration are vital to the success of learning, especially when learning is happening remotely $[13,17]$.

\subsection{Playful education in times of crisis}

The COVID-19 emergency has made educational institutions worldwide go from onsite/offline to online teaching. This crisis has required school institutions, some of them reluctant to change, to accept modern technology and change their educational approach. However, many problems and challenges are associated with online teaching and learning $[1,6,15]$. Online learning implementation requires robust platforms, reliable internet access, and working devices for teachers and students, which often cannot be given for granted $[1,13]$. Many different technologies are available for online education - such as video conferencing tools, online whiteboards, productivity software; yet, all require specific expertise and provide technical and pedagogical challenges to their user at multiple levels 
(teachers, students, and families alike) [6,17]. Especially for younger students, online teaching can be perceived as boring and disengaging [6]. Moreover, online learning requires specific skills such as basic digital literacy, effective time-management, persistence, and independence above all $[1,6,13]$. Major barriers for online learning as perceived by students are the lack of community and social connection, technical problems, and difficulties in understanding instructional goals [15]. Given these reasons and considering the current emergency situation, online learning might inhibit the social experience offered by onsite activities and ultimately widening educational inequalities $[1,13]$.

In Italy, the first European country hit by the pandemic and where this study took place, schools and universities closed on the 5th of March 2020 and implemented distance learning for the rest of the $2019 / 2020$ school year. It is important to note that in Italy the school closure was part of a government decree that essentially prohibited all movements of people within the whole territory and the closure of all non-essential business activities. While school closures present an effective solution to enforce social distancing and thus limiting the spreading of the pandemic, prolonged closures tend to have a disproportionately negative impact on students [12]. Out of school, students have fewer learning opportunities and chances to socialize, while parents may face greater challenges in balancing work and family duties when their support in homeschooling is required. In this respect, technology might represent an effective means for supporting community engagement and communication $[1,6,9]$.

Generally speaking, research shows that the Italian school system demonstrated a reasonable maturity and robustness in terms of technological infrastructure [10]. Nevertheless, some teachers tended to use technologies to reproduce traditional transmissive teaching dynamics [10], showing a certain reluctance or inability to adapt their teaching approaches to the new conditions of learning. Moreover, the emergency affected also students' lifestyle: daily routines changed during the quarantine, and children spent more time using screens, less time doing physical activity, and sleeping more hours [16]. Parents reported a number of negative psychological effects on their children, such as increased boredom, irritability, restlessness, nervousness, and feelings of loneliness [16]. To mitigate these negative effects, the school community has enacted strategies to cope with the new situations, rethinking their practice and stimulating innovation [7]. These new practices include also innovative approaches to teaching, such as the use of playful and gameful education. In this respect, playful education can be a valuable support to face the challenges imposed by the COVID-19 emergency. Recent meta-reviews $[3,11]$ pointed out the ability of gamification to motivate students to perform learning tasks, fulfil their need for recognition, and provide relevant feedback on their individual or group work. However, the majority of studies investigate the application of gamification in formal education, mainly considering in-class settings. In this paper, we present an experience with a gamified system originally developed for in-class activities related to sustainable mobility and then converted to online use when students were forced to attend school remotely because of the COVID-19 pandemic. Through the evaluation conducted at the end of the school year, we investigated the efficacy of the adapted version of the system in promoting family and school community engagement and how its perception and use changed with respect to the previous in-class setting during the health emergency.

\section{KIDS GO GREEN: PLAYFUL EDUCATION AND SUSTAINABLE MOBILITY}

Kids Go Green (https://kidsgogreen.eu/en/) is a web-based platform designed for children (aged 6-14) that promotes sustainable and active mobility by transforming school commuting and daily trips into an educational and playful collective experience, involving the entire school community (children, teachers, and families) in a gameful educational journey. In the original version of the platform, sustainable kilometers made by each child on their home-school trips (e.g., on foot, by bike, with the school bus) contribute to the progress of the whole class on a virtual educational journey to places in the real world that is personalized according to the group interests and capabilities $[8,14]$. As the journey progresses, a digital map shows the accomplishments of the class and the arrival to intermediate stops (see Figure 1). The platform supports teachers to plan the thematic journey and associate multimedia educational material to each stop, making each achievement an opportunity for in-class learning. Moreover, the platform includes mechanisms to dynamically adjust and calibrate the game parameters, providing class-level and school-level challenges for sustaining participants' motivation. Since 2016, Kids Go Green has been used by more than 2900 students in 27 school communities in Italy to encourage students and families to adopt more sustainable solutions in homeschool trips. During the years, Kids Go Green demonstrated a strong impact in terms of increased awareness by the children and their families on the mobility habits also beyond the conclusion of the trials, confirming a significant impact of the initiative in promoting a collective change towards more sustainable mobility habits [14].

\subsection{From sustainable mobility to good deeds}

Kids Go Green was used by 17 schools in Italy (950 students aged from 7 to 12) when the COVID-19 emergency started to spread in Europe. The health emergency hit Italy particularly hard, leading the Italian government to order the closure of all schools nationwide in March 2020 for the remaining school year to contain the virus outbreak. In this scenario, Kids Go Green has been adapted by revising its main game metaphor to become a way to support distance learning in the new context.

After an initial suspension of a few weeks, several teachers expressed their willingness and need to use Kids Go Green as a supporting tool for distance learning. Among the main reasons supporting this choice we can highlight (i) the serious difficulties teachers experienced in keeping the group relationships of their classes alive and effectively manage distant learning; (ii) the significant fatigue expressed by families in keeping their children interested and motivated in distance learning activities during the lockdown period. Consequently, the Kids Go Green project team decided to co-design and develop some extensions to the platform that could be exploited by the school community in this new and complex educational and social context. The first line of action concerned the adjustment of the game modes and tools to support its operations also during remote teaching. In particular, together with teachers, we co-designed and developed new approaches that allow pupils to 

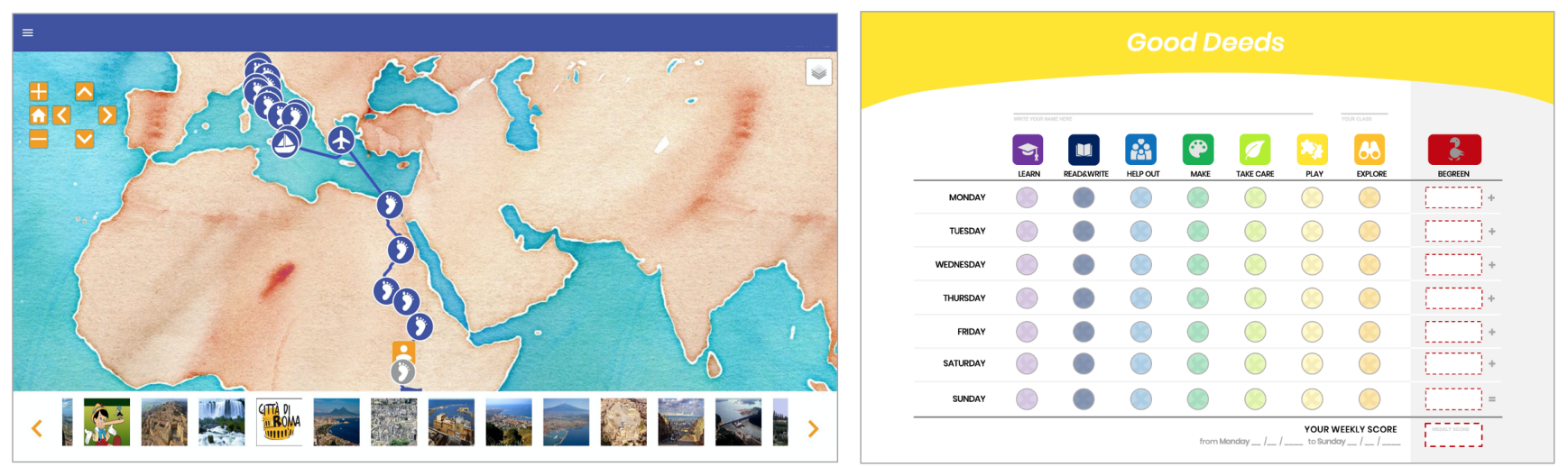

Figure 1: Screenshots from Kids Go Green application. World map and virtual journey (left) and form for reporting the "good deeds" performed by the children (right).

advance as a group in the Kids Go Green game reporting the "good deeds" performed at home.

The original platform encouraged students to report the sustainable distances made on their trips from home to school (e.g., on foot, by walking bus, by bike) - the so-called "green distances" - which then would be spent to advance in a virtual educational journey (Figure 1, left). The first element subject to the intervention had to concern the advancement modality of children in the virtual journeys. Since during the school closure, home-school trips were no longer an option, the gaining of virtual points was associated with a series of "good deeds" that students could carry out at home. Similarly to the "green distances", these points would then be summed up to allow the entire class to progress in the virtual journey and to unlock the multimedia materials to be used by the teachers in their distance learning activities.

The good deeds, identified by the project team together with teachers, covered a number of areas and were selected for valuing self-esteem, responsibility, independence and active commitment in children. Specifically, they were categorized as: Learn (e.g. doing the homework, following online lessons), Play (e.g. playing games, practicing exercise activities, dancing), Explore (e.g. watching a documentary, making a science experiment), Help out (e.g. helping out in recycling the waste, cleaning up), Read\&Write (e.g. reading a story, writing a diary entry, writing to a friend), Take care (e.g. watering plants, taking care of pets) and Make (e.g. drawing, composing a song, acting).

The adapted version included also changes in the way students could report their contributions to the virtual journey. In the original version of Kids Go Green, during the first lecture of the morning, students together with the teacher collectively filled a form using an interactive whiteboard by specifying how they reached the school that day and reporting the "green distances" travelled. Conversely, during the school closure, students were asked to record the actions carried out day by day by filling a form through two alternative methods: on a paper form sent weekly to the teachers as picture attachment via email or WhatsApp, or on an online form accessible through PCs, tablets, or smartphones (Figure 1, right). Students were free to fill in the form whenever they wanted but, compared to the original method, the activity was mainly individual and asynchronous. Similarly to the original platform, the good deeds were used by the class to advance in the virtual journey created by their teachers. Each virtual journey included a number of different stops and at each stop a different set of multimedia resources (including videos, documents and activities) were unlocked and presented to the class.

\section{THE STUDY: KIDS GO GREEN DURING LOCKDOWN}

Since the school year 2019-2020 was severely affected by the COVID19 pandemic, the "standard" evaluation of Kids Go Green that has been carried out over the past years was revised to meet the new requirements and operational context. The evaluation aimed at exploring how children and their families experienced the new modalities implemented in the "good deeds" version of the Kids Go Green campaign also with respect to the previous version based on sustainable mobility. Specifically, we aimed at exploring three main topics: (1) children's engagement in the Kids Go Green campaign, their understanding and use of the different gamification mechanisms for collecting points, and their suggestion for future improvements; (2) parents' engagement in the initiative and how they perceive the motivation and involvement of their child(ren) before and during the lockdown period; (3) teachers' motivation, satisfaction, and perception of the Kids Go Green initiative and its implementation.

\subsection{Methodology and Data Collection}

The evaluation utilized a mixed-method approach. Quantitative data, collected via the questionnaires and the analysis of the log files, enabled us to collect information on the perceived value of Kids Go Green and the actual use of the platform. Qualitative data, collected via open-ended questions in the surveys and from the workshops, enabled us to obtain an in-depth understanding of the users' experience of the platform before and during the school closure. 
For primary schools, questionnaires were digitally administered to 84 families involved in the project. The questionnaire was divided in two parts: a section for parents and a dedicated section for primary school children to be filled out by the children themselves with the assistance of their parents (if needed). Some questions were left open to allow respondents to better explain their preferences or leave comments. Questions for parents investigated their knowledge and interest about Kids Go Green, while questions for children investigated their experience, exploring what they liked the most and the least of Kids Go Green in general.

For middle schools, questionnaires were administered to 69 middle school students involved in the project. Open-questions for middle school students revolved around: what students liked the most and the least of Kids Go Green in general; if and why their level of engagement had changed when the "good deeds" version was introduced; what features they liked the most comparing the two versions and why; and suggestions for improvements. These personal pieces of text have been analyzed through Thematic Analysis [5]. In addition to the survey, an online workshop was organized to explore how students perceived the change between the two modalities and how they compared the two experiences. The workshop involved two classes with a total of 28 students (16 F, $12 \mathrm{M}$, 11-12 years old) and two teachers, who participated on a voluntary basis during the class hours. After an initial presentation of the workshop's topics to all the participants by three researchers, students were divided by their teachers into three different groups. Each researcher was assigned to a group of 9-10 children, while the two teachers could freely move across each online meeting room. During the workshop students were engaged in an open discussion facilitated by the use of a collaborative whiteboard (Google Jamboard) for reporting their experience with the initiative and for comparing the two versions of the platform.

Finally, we administered a dedicated questionnaire to 7 teachers involved in the initiative (4 primary school teachers and 3 middle school teachers) in order to explore their opinions about the use of Kids Go Green in the two versions, before and after the lockdown.
In the following sections, we present the outcomes of this evaluation, starting with the analysis of the logs and then reporting results from the perspective of parents, students and teachers.

\section{RESULTS}

\subsection{Log Analysis}

In the period from the national lockdown (March 2020) to the end of the school year (June 2020), 15 classes and 275 students took part in the Kids Go Green "good deeds" initiative. A total of 8 virtual journeys have been created with an average number of 10 stops for each journey (for a total of 84 stops). During the initiative, students reported a total of 12.533 good deeds. Specifically, the number and percentage of good deeds performed in each category were as follow (in descending order): Learn (2378, 19\%), Play (2360, 18.8\%), Help out $(2204,17.6 \%)$, Read\&Write $(1828,14.6 \%)$, Take care $(1404,11.2 \%)$, Make (1321, 10.5\%), Explore (1040, 8.3\%).

\subsection{Parents' perspective}

At the end of the school year 2019/2020, most families were aware of the Kids Go Green initiative: $66 \%$ (56 out of 84 respondents) of parents reported to have a very high (36\%) or high (30\%) knowledge of the initiative. $29 \%$ reported to have a moderate knowledge, while $5 \%$ and $1 \%$ reported low or very low scores respectively. Furthermore, parents' satisfaction with the Kids Go Green initiative was very high $(30 \%)$ or high $(41 \%)$ in the majority of cases $(71 \%)$, confirming what has been observed in previous editions [14]. In Figure 2, we present parents' perception of the influence that the Kids Go Green initiative had on their children. Parents perceived a positive influence in all investigated aspects. The highest positive influence was observed in encouraging students to do "good deeds" at home (post-lockdown version) (52\% of responses on the higher end of the scale, i.e. "Extremely" and "Very much"), in promoting sustainable mobility habits (pre-lockdown version) (61\%), and in its didactic value, both in terms of ensuring continuity of didactic activities (53\%) and facilitating innovation in didactic (52\%). Kids Go Green impact was perceived to be very relevant also for children

\section{HOW MUCH DID KIDS GO GREEN POSITIVELY INFLUENCE THE FOLLOWING ASPECTS?}

Extremely $\square$ Very much $\square$ To some extent $\backsim$ A little $\quad$ Not at all

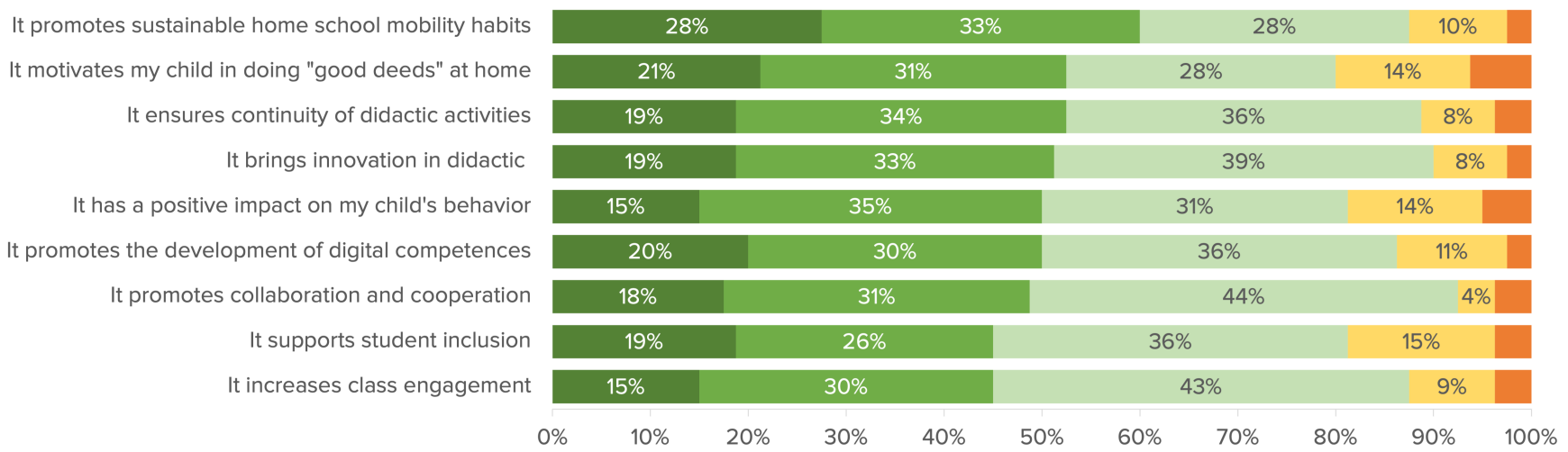

Figure 2: Parents survey - Positive influence of Kids Go Green. 

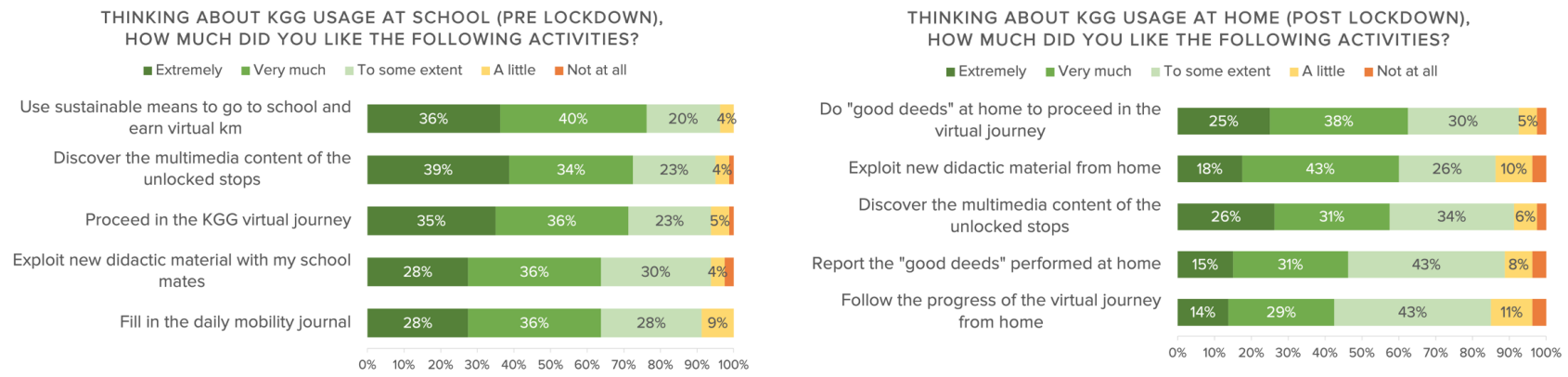

Figure 3: Children survey - Appreciation of different activities: pre-lockdown experience (left) and post-lockdown experience (right)

acquisition of digital competencies (50\%), student collaboration and cooperation (49\%), student inclusion (45\%), and for maintaining class-level relationships (45\%). Some comments by parents in the open questions support these findings: "Now my child is much more careful about recycling, whereas he did not care at all before", "My child is much more motivated to help in the housework", "She has been very careful to the small things like diminishing the use of plastic, keeping everything tidy, and helping in the housework".

To compare the two versions of Kids Go Green, we asked parents whether they perceived a change in the motivation of their child in participating in the initiative. The majority of parents (56\%) reported that they perceived an increase in motivation of their child with Kids Go Green "good deeds" while only $10 \%$ reported a decrease (for 23\% the motivation remained unchanged, 11\% cannot say). Parents' perception of the increased motivation probably relates to the reported easiness of use of the "good deeds" version by children ("It is easier for the children to manage autonomously with the good deeds version"). Nevertheless, besides its good performance in keeping children motivated and stimulated about the topic of sustainability at home, the adapted platform is perceived to be less engaging than the sustainable mobility version because of the lonely condition when doing the tasks and enjoying the contents ("The collaboration with school mates made Kids Go Green sustainability more engaging"; "It looked like children were not pursuing a common goal. Therefore, even if in practice they kept doing the good deeds, they lost interest in reporting them online. Reporting everybody's contribution in class has for sure an added value"; "Counting kilometers in the sustainability version is more immediate and engaging because the children do it all together").

\subsection{Primary and middle school students' perspective}

The second section of the questionnaire had to be completed by the children themselves ( $\mathrm{n}=153$ of which 84 primary school, 69 middle school). The items of this part of the questionnaire aimed to evaluate and compare the children's experience with Kids Go Green in its two versions: pre- and post-lockdown. We report the results in Figure 3 (left), for the original pre-lockdown Kids Go Green version, and in Figure 3 (right), for the "good deeds" post-lockdown version.
Despite the parents' perception, it emerged that children, although appreciating both versions, reported more involvement in the original pre-lockdown version of Kids Go Green. In particular, they enjoyed more filling in the daily mobility journal than the "good deeds" form (Wilcoxon signed test: $W=2730, p<.001$ ). They preferred observing the progress on the journey in class instead of following it from home $(W=4436, \mathrm{p}<.001)$, as well as discovering $(\mathrm{W}=1400, p<.001)$ and enjoying $(W=1980, p<.001)$ unlocked multimedia content with their schoolmates instead of unlocking them while being at home. No significant differences were observed between contributing by adopting sustainable mobility habits than by doing good deeds at home.

The analysis of the open ended questions and results from the workshop revealed that children did not like the repetitiveness and redundancy in the interaction with the "good deeds" version of Kids Go Green: "I did not like the daily journal because the goals were always the same"; "I did not like the fact that in 'good deeds' we need to fill in the journal everyday with the same actions and there is no way to add a personalized deed... so after a while it became repetitive and boring". Some of the criticisms related to the loneliness of the interaction rather than to some lack in the interface and so were caused by the situation of being forced to stay at home and not to be physically with their school friends: "to watch the videos on my own, to make the roll call on my own..."; "I do not like as much doing it from home, but still, I enjoy it".

\subsection{Teachers' perspective}

Teachers who responded to the questionnaire $(n=7)$ were generally satisfied with their experience with both versions of Kids Go Green ( $\mathrm{n}=4$ "a lot", 1 "very much", and 2 "quite a bit"). All teachers think that Kids Go Green helped them in bringing innovation in the didactic activity (2 "a lot", 4 "very much" and 1 "quite a bit") and contributed in promoting students' development of digital skills ( 1 "a lot", 5 "very much" and 1 "quite a bit"). They also perceive a positive impact with respect to their own digital competencies ( 1 "a lot", 4 "very much", 1 "quite a bit" and 1 "a little"), and on students' inclusion ( 2 "a lot", 3 "very much", 1 "quite a bit" and 1 "a little") and class relationships (1 "a lot", 1 "very much" and 5 "quite a bit"). A positive but more limited impact is perceived also 
in terms of students' behavior (1 "a lot", 1 "very much" and 5 "quite a bit") and didactic continuity (1 "a lot", 1 "very much" and 5 "quite a bit"). Considering teachers' experience when switching to the "good deeds" version of Kids Go Green, teachers reported that Kids Go Green has motivated students during the national lockdown (1 "a lot", 4 "very much" and 2 "quite a bit"). They also reported how Kids Go Green supported the organization of distance learning (1 "a lot", 4 "very much", 1 "quite a bit" and 1 "a little"), promoting continuity in didactic activities (1 "a lot", 4 "very much", 1 "quite a bit" and 1 "a little"), and contributing to maintain relationships among students ( 1 "a lot", 4 "very much", 1 "quite a bit" and 1 "a little").

Concerning the perceived change in terms of motivation, comparing the two versions of Kids Go Green, teachers reported mixed views: 3 teachers reported that students' motivation increased with Kids Go Green "good deeds", while other 3 said that the motivation diminished (one teacher did not report any change). This difference in the teachers' experience is also reported in terms of the capability of Kids Go Green "good deeds" to involve a lower number of students than the traditional version (reported by 3 of them). Analysis of the open questions revealed that during distance learning several students were "difficult to reach or not reachable at all". Teachers reported that for some students distance learning and online activities constituted a barrier to their engagement in all didactic activities, including the Kids Go Green initiative.

\section{DISCUSSION}

In this study, we evaluated how students, parents, and teachers perceived two versions of the Kids Go Green platform through a mixed-method approach and we learned aspects that worked well and those that failed to reach their potential.

The first positive aspect was the process of adaptation to the new educational and social context in response to the health emergency. The Kids Go Green "good deeds" version, that was rapidly co-designed with teachers during the first weeks of the national lockdown, successfully provided an additional instrument for supporting distance learning and making online activities more engaging for students. The fact that both teachers and students were already acquainted with the platform played an important role in reducing the friction that characterise the adoption of new teaching instruments and communication modalities in the school setting. The other positive aspect was the involvement and satisfaction of families, who became important stakeholders of the school community during the lockdown period. Parents increased their awareness about Kids Go Green and reported a positive influence of the platform in encouraging their children to perform "good deeds" at home. It is interesting to note that all seven types of good deeds were reported by students in a similar amount, meaning there was a good balance between all types of good actions.

More nuanced results are those related to students' motivation in using the adapted version. Parents reported an increase in their children's motivation, but it should be mentioned that they were less aware of how their children used Kids Go Green when the activity was carried out in class, while they became more involved with the introduction of the good deeds. Students generally reported to be less engaged in the "good deeds" version. In this case, it is worth noticing that most of the students' comments collected through the questionnaires and the workshops referred to the extremely difficult situation of distance learning against which even an appreciated platform could not eliminate the lack of physical contact with their schoolmates that characterized the lockdown period [13, 17]. Teachers provided a third point of view concerning students' motivation and their use of the platform: almost half of the teachers claimed the motivation increased while the other half claimed it decreased. However, their answers to the open questions point to the fact that some students were unreachable during lockdown and this was independent of the communication means but was due to general barriers to distance learning.

The results also provide insights on aspects that can be improved to better support student engagement and involvement while being at home, including personalization and different ways for promoting children's active role when interacting with the platform [18]. Students reported how the platform was more engaging when they were at school than while being at home. More precisely, several aspects of the experience changed between the two versions and could be further improved in the "good deeds" version such as the reporting of the good actions, the advancement on the map and the fruition of the educational material. The reporting of good deeds was made available via a Google form because it was faster to implement under time pressure. However, it allowed reporting the good deeds only by checking a "Done!" flag under one of the seven generic categories of good deeds. Students expressed the lack of a free text field for describing in more detail the good deed made, showing the willingness to better describe or document their activities. With a more detailed description of the good deeds, it is possible for classmates to take inspiration from others' deeds and for the teachers to start discussions with the whole class.

In general, the reduced student motivation with the "good deeds" version can be explained by the general social isolation that students experienced during the lockdown [13, 17]. Before the lockdown, the Kids Go Green activities took place in the class where all the students were physically present while the adapted version had to be used from home. However, there are still some opportunities for further increasing the sense of belonging and shared presence. For example, the advancement of the virtual journey on the map, that now is represented asynchronously, can be made synchronous and experienced in groups. Similarly, reaching a stop in the journey and unlocking the educational content can be made synchronous to support a shared and collective experience among classmates. Moreover, as already suggested above, also seeing the good deeds performed by classmates since the last lesson could be a shared activity to foster discussion and mutual inspiration.

In conclusion, the main suggestion that emerged from this evaluation is to enhance the functionalities of the platform in order to improve the sense of shared presence and collective actions that are typical when students are in presence at school but that is what lacked when students were remotely connected from their houses. This is relevant also in view of possible future school closures or for hybrid learning strategies (i.e., alternation of face-to-face and remote teaching). 


\section{CONCLUSION AND FUTURE WORK}

With this work, we present a case study of the adaptation of a playful education platform (Kids Go Green) from in-class to online usage. The platform was originally conceived for motivating students in reaching school with sustainable transportation means. Then, following the school closures and the massive adoption of distance education caused by the 2020 COVID- 19 health emergency, the platform was adapted to accommodate the new situation by inviting students to perform good deeds at home. The evaluation of the adapted platform has shown overall good results in providing a positive experience to all members of the school community (students, teachers, parents) and in keeping them engaged, even though it was not able to overcome the generalized feeling of isolation. We will continue investigating this topic also for the school year 2020/2021 and future work will provide a thorough and systematic analysis of the quantitative and qualitative data we collected. We believe that exploring how educational platforms can be made flexible and be adapted to hybrid learning approaches that combine remote and in classroom learning is useful to support activities during school reopening and in preparation for potential resurgence.

Moreover, this hybrid way to interact and collaborate at school over a distance may be useful also in other situations, not only in the context of emergency teaching. For example, to connect classes with students who cannot attend school for a certain period of time because of health issues or to promote collaborations between different schools, such as in school twinning and partnership projects between institutes.

\section{ACKNOWLEDGMENTS}

This work is partially supported by Fondazione VRT within the 'Kids Go Green - Ripartiamo insieme!' project

\section{SELECTION AND PARTICIPATION OF CHILDREN}

Children and their parents responded to the questionnaire on a voluntary basis and the questionnaire was anonymous. The family questionnaire included a section for parents and a dedicated section for their children. The questionnaire for middle school students was administered by their teachers. The workshop was scheduled during the participants' school day together with their class teachers as part of their regular teaching. Teachers were also allowed to attend the whole activity session.

\section{REFERENCES}

[1] Olasile Babatunde Adedoyin and Emrah Soykan. 2020. Covid-19 pandemic and online learning: the challenges and opportunities. Interactive Learning Environments 0, 0 (Sept. 2020), 1-13. https://doi.org/10.1080/10494820.2020. 1813180
[2] Zaheer Allam and David S. Jones. 2020. On the Coronavirus (COVID-19) Outbreak and the Smart City Network: Universal Data Sharing Standards Coupled with Artificial Intelligence (AI) to Benefit Urban Health Monitoring and Management. Healthcare 8, 1 (March 2020), 46. https://doi.org/10.3390/healthcare8010046

[3] Shurui Bai, Khe Foon Hew, and Biyun Huang. 2020. Does gamification improve student learning outcome? Evidence from a meta-analysis and synthesis of qualitative data in educational contexts. Educational Research Review 30 (June 2020), 100322. https://doi.org/10.1016/j.edurev.2020.100322

[4] Nicola Luigi Bragazzi, Haijiang Dai, Giovanni Damiani, Masoud Behzadifar, Mariano Martini, and Jianhong Wu. 2020. How Big Data and Artificial Intelligence Can Help Better Manage the COVID-19 Pandemic. International fournal of Environmental Research and Public Health 17, 9 (Jan. 2020), 3176. https://doi.org/ 10.3390/ijerph17093176

[5] Victoria Clarke, Virginia Braun, and Nikki Hayfield. 2015. Thematic analysis. In Qualitative psychology: A practical guide to research methods, J. A. Smith (Ed.). SAGE, 222-248.

[6] Shivangi Dhawan. 2020. Online Learning: A Panacea in the Time of COVID19 Crisis. Journal of Educational Technology Systems 49, 1 (Sept. 2020), 5-22. https://doi.org/10.1177/0047239520934018

[7] Viv Ellis, Sarah Steadman, and Qiming Mao. 2020. 'Come to a screeching halt': Can change in teacher education during the COVID-19 pandemic be seen as innovation? European fournal of Teacher Education 43, 4 (Aug. 2020), 559-572. https://doi.org/10.1080/02619768.2020.1821186

[8] Elisabetta Farella, Michela Ferron, Davide Giovanelli, Chiara Leonardi, Annapaola Marconi, Paolo Massa, Amy L. Murphy, Michele Nori, Marco Pistore, and Gianluca Schiavo. 2020. CLIMB: A Pervasive Gameful Platform Promoting Child Independent Mobility. IEEE Pervasive Computing 19, 1 (Jan. 2020), 32-42. https://doi.org/10.1109/MPRV.2019.2939730

[9] Dana Rose Garfin. 2020. Technology as a coping tool during the coronavirus disease 2019 (COVID-19) pandemic: Implications and recommendations. Stress and Health 36, 4 (2020), 555-559. https://doi.org/10.1002/smi.2975

[10] Carlo Giovannella, Marcello Passarelli, and Donatella Persico. 2020. The effects of the Covid-19 pandemic on Italian learning ecosystems: The school teachers' Perspective at the steady state. Interact. Des. Archit. 45 (2020), 264-286.

[11] Christopher Groening and Carmen Binnewies. 2019. "Achievement unlocked!" - The impact of digital achievements as a gamification element on motivation and performance. Computers in Human Behavior 97 (Aug. 2019), 151-166. https: //doi.org/10.1016/j.chb.2019.02.026

[12] Megan Kuhfeld, James Soland, Beth Tarasawa, Angela Johnson, Erik Ruzek, and Jing Liu. 2020. Projecting the Potential Impact of COVID-19 School Closures on Academic Achievement. Educational Researcher 49, 8 (Nov. 2020), 549-565. https://doi.org/10.3102/0013189X20965918

[13] Wim Van Lancker and Zachary Parolin. 2020. COVID-19, school closures, and child poverty: a social crisis in the making. The Lancet Public Health 5, 5 (May 2020), e243-e244. https://doi.org/10.1016/S2468-2667(20)30084-0

[14] Annapaola Marconi, Gianluca Schiavo, Massimo Zancanaro, Giuseppe Valetto, and Marco Pistore. 2018. Exploring the world through small green steps: improving sustainable school transportation with a game-based learning interface. In Proceedings of the 2018 International Conference on Advanced Visual Interfaces (AVI '18). Association for Computing Machinery, New York, NY, USA, 1-9. https://doi.org/10.1145/3206505.3206521

[15] Lin Y. Muilenburg and Zane L. Berge. 2005. Student barriers to online learning: A factor analytic study. Distance Education 26, 1 (Jan. 2005), 29-48. https: //doi.org/10.1080/01587910500081269

[16] Mireia Orgilés, Alexandra Morales, Elisa Delvecchio, Claudia Mazzeschi, and José P. Espada. 2020. Immediate Psychological Effects of the COVID-19 Quarantine in Youth From Italy and Spain. Frontiers in Psychology 11 (2020). https: //doi.org/10.3389/fpsyg.2020.579038

[17] Russell M Viner, Simon J Russell, Helen Croker, Jessica Packer, Joseph Ward, Claire Stansfield, Oliver Mytton, Chris Bonell, and Robert Booy. 2020. School closure and management practices during coronavirus outbreaks including COVID-19: a rapid systematic review. The Lancet Child \& Adolescent Health 4, 5 (May 2020), 397-404. https://doi.org/10.1016/S2352-4642(20)30095-X

[18] Guanghai Wang, Yunting Zhang, Jin Zhao, Jun Zhang, and Fan Jiang. 2020. Mitigate the effects of home confinement on children during the COVID-19 outbreak. The Lancet 395, 10228 (March 2020), 945-947. https://doi.org/10.1016/ S0140-6736(20)30547-X 